\title{
Imaging Findings of Bisphosphonates-Associated Osteonecrosis of Jaw: Literature Review and Report of 3 Cases
}

\author{
Lazar Adela Cristina1, Hedesiu Mihaela², Ilea Aranka¹, Baciut Grigore ${ }^{3}$, Pacurar Mariana ${ }^{4}$, Campian \\ Radu-Septimiu ${ }^{1}$
}

\author{
1 Department of Oral Rehabilitation, Oral Health and Dental Office Management, "Iuliu Hațieganu" University of Medicine and Pharmacy Cluj-Napoca, Romania \\ 2 Department of Dental Radiology, "Iuliu Hațieganu" University of Medicine and Pharmacy Cluj-Napoca, Romania \\ ${ }^{3}$ Department of Oral and Maxillofacial Surgery, "luliu Hațieganu" University of Medicine and Pharmacy Cluj-Napoca, Romania \\ ${ }^{4}$ Faculty of Dental Medicine, University of Medicine and Pharmacy Targu-Mures, Romania
}

\begin{abstract}
Purpose: The present study aims radiological aspects of the occurrence of osteonecrosis of the jaw age groups both in receiving i.v bisphosphonates tratment Imaging assessment of bisphosphonates therapy-induced osteonecrosis of the jaw it is important to differentiate neoplastic invasion, osteomyelitis, osteoradionecrosis induced by radiation or bone related pathology of general diseases.

Material and method: We conducted a retrospective clinical study including 22 patients (8 men and 14 women) with various stages of osteonecrosis of the jaw.

Results: Radiological examinations using CBCT are required in all therapeutic approach of osteonecrosis of jaw cases providing accurate informations of position, dimension and the link with anatomical structures.Our study showed that the prevalence of osteonecrosis of mandibular growth is higher in women than in men and the risk of osteonecrosis of the jaw in appearance is depending on age factor witch occurs more often between age 52-59 and 73-80 years old.

Conclusion: The multitude of complications due to treatment with bisphosphonates bind to an early and specialized therapeutic approach. Radiological examinations is a first choice in the detection and early diagnosis of osteonecrosis of the jaw, patients requiring a permanent supervision by the physician and dentist.
\end{abstract}

Keywords: bisphosphonates osteonecrosis of the jaw, CBCT, osteolysis, thickening lamina dura

Received 8 December 2015 / Accepted: 04 June 2015

\section{Introduction}

Bisphosphonates represents structural analogues of pyrophosphates used in tratment of osteoporosis (Liberman et al.,1995; Mortensen et al.,1998) [1], Paget disease, myeloma solid bone metastasis (Berenson,1996; Hortobagyi et al., 1996; Major, 2002) [2], patients receiving high doses of corticoises [3] and according to recent studies can be used even on children with osteogenesis imperfecta. They inhibit bone resoption and are absorbed to mineral surfaces in bone where they interfere with the action of the osteoclasts, being ineternalised by osteoclasts and intersect with specific biochemical processes.

Long term use of bisphosphonate suppress bone turnover to the point that such microdamage persists and accumulates. The result is hypodynamic bone with decreased biomechanical competence [4]. Alongside with their properties and benefits, bisphosphonates can generate side effects such as burning sensation and blisters in the oral cavity, erosive oesophagitis associated with painful difficult swallowing, oesophagal stenosis, uveitis, gastric ulcerations and abdominal pain esophageal mucosal ulceration. When the treatment is carried out intravenously (i.v.) the following symptoms can be also present in addition: pseudo-flue

* Correspondence to: Mariana Pacura

E-mail: marianapac@yahoo.com syndrome (consisting in fever, shivering, arthralgias, myalgias, bone pain), fatigue, phlebitis, anemia, weakness, edemas or dyspnea [5-7].

In oro-maxillo-facial area, nitrogen containing bisphosphonates administrated mostly intravenously can induce osteonecrosis of the jaw bone(BRONJ) and is identified as 'jaw avascular bone necrosis', witch can appear as a growing, painful, unilateral swelling of the mandible in patients receiving bisphosphonates [8]. According to definition, BRONJ is characterised by the presence of an un-healing wound in the maxillofacial region with bone exposure, more than 8 weeks after dental surgery. It is characterized by tissue dehiscence, chronic bone devitalization, hypocellularity, lytic radiologic $[9,10]$ infection, possibly with purulence, altered sensation (eg, numbness or heavy sensation) [11]. First described in 2003, BRONJ is generally defined by the presence of transmucosal or transcutaneous jawbone exposure for at least eight weeks, a history of bisphosphonate administration, and the absence of any history of irradiation to the head and neck region [12-14]. The blood supply is more expressed at the level of the jaw as compared with other bone structures. At the same time, as a consequence of the mastication process and the mechanical stimulation produced by the teeth, the jaw present a significantly faster process of bone remodelling. All these, together with the fact that the arteries irrigating the jaws 
are terminal arteries, could explain the higher risk of necrosis encountered at this level. At the same time, the necrotic bone carries a higher risk of associated infections, deriving from the fact that mucosal layer that separates them from the oral cavity is very thin [11]. Despite oral administrated bisphosphonates may cause oral mucosal lesions (apparently arising from direct contact injury).

We focus our review on bisphosphonate-associated osteonecrosis of the jaw from clinically and radiologically point of view as well as the presentation of three cases with BRONJ. As a literature data base, in 2003, Marx described 36 cases of exposed necrotic jaw bone detected in patients who had been treated with intravenous bisphosphonates as part of cancer therapy. 78\% of the cases of painful exposure of the jaw bone occurred after dental extractions, but $22 \%$ were spontaneous (Marx 2003) [9]. In the following table, it is ilustrated the 4 main stages of BRONJ with it's clinical signs.

Table I. Clinical staging of bisphosphonate-related osteonecrosis of the jaw according to Wilde et al. 7

\begin{tabular}{cc}
\hline Stage 0 & No exposed necrotic bone \\
\hline Stage 1 & Asymptomatic exposed necrotic bone or single intraoral fistula \\
\hline Stage2 & Exposed necrotic bone associated with pain and infection \\
\hline Stage 3 & $\begin{array}{c}\text { Exposed necrotic bone associated with pain; infection with } \\
\text { swelling and abscesses; multiple intraoral fistulas and ex- } \\
\text { tended osteolysis in the radiology findings }\end{array}$ \\
\hline Stage4 & $\begin{array}{c}\text { Exposed necrotic bone associated with pain; infection with } \\
\text { swelling and abscesses; pathological fracture, naso-oral } \\
\text { fistula, extraoral fistula, or osteolysis extending to the inferior } \\
\text { border }\end{array}$ \\
\hline
\end{tabular}

For a clear and precise diagnosis radiographs should be performed to certify the presence of BRONJ.Current studies show the need for radiological findings in BRONJ and also provides data as case reports or case series, in whitch are described different stages of BRONJ in patients with concomitant illness [15-17]. There are several types of X-rays used for clear and precise diagnosis of BRONJ.

Plain Film Radiography Thus, among the most commonly used in current dental practice is plain radiography film. In this category falls retro film and orthopantomography (OPT, panoramic X-ray), which provides data about thickening of the lamina take, osteosclerosis, osteolysis, diffuse sclerosis, dense woven bone, subperiosteal bone deposition, failure of post-surgical remodelling, poor healing or nonhealing of the extraction sites [18-20]. Clinic association between signs and radiological stage BRONJ showed that initially stage presents with sclerosis at the alveolar margin with thickening of the lamina last. As BRONJ evolves, there seems to be an increase in the degree of sclerosis. Stage 3 and 4 show radiographic evidence the narrowing of the mandibular canal and clinically patients may have signs of paresthesia [21].

Computed Tomography In the last years, CBCT have become increasingly popular in oral-maxillofacial surgery. A big advantage of CBCT is the access to 3-dimensional (3D) of bone structures of very high resolution. Exposure to the radiation is higer than OPT, but seems lower than Multisliced Computer Tomography (MSCT) [18]. Computer Tomograph is an indispensable diagnostic and radiological method for BRONJ. CT scan provides accurate data, which in many cases may seem confusing or unclear on a plain film radiography. A corelation between the sensitivity of Computer Tomograph and panoramic $x$-rays in the diagnostic evaluation of $\mathrm{BRONJ}$ indicated that panoramic $\mathrm{x}$-rays tend to underestimate the extent of lesions and miss the presence of small sequestra [21]. CT findings shows that focal sclerosis is present in early stages with the presence of disorganized, trabecular pattern and poor corticomedullary differentiation. Affected bone site shows total bone involvement. As the disease advances, periosteal reaction and sequestration occur.The CT findings for BRONJ also include cervical lymphadenopathy,which is related to the presence of infection in the exposed bone [21].

\section{Material and Method}

We conducted a retrospective clinical study in the MaxiloFacial Surgery Clinic Cluj-Napoca including 22 patients (8 men and 14 women) with various stages of osteonecrosis of the jaw between 2010-2012. Clinical signs showed exposed necrotic bone associated with pain and infection, extra oral fistulas and signs of osteolysis extending to the inferior cortical border. Radiological findings showed lamina dura thickening, osteolysis, encroachment on the mandibular canal, sequestrum. Examinations were made by specialist radiologist, oncologist and nurse radiology using Newton unit 3GQR Italy performing sagittal and axial sections with a detector field (FOV) of 12". The images resulted in CBCT were viewed and processed using NNT softwer. The following 3 cases were part of this retrospective study:

\section{Case 1}

A woman aged 44 with neoplasic breast cancer and under bisphosphonate therapy (Alendronate and Goserelin Acetate) developed a Stage 4 of BONJ (Exposed necrotic bone associated osteolysis extending to the inferior border) located on the lateral left side of the mandibular arch. The radiological protocol of the $\mathrm{CBCT}$ included the following data: axial thicknes $0,500 \mathrm{~mm}, \mathrm{FSV}: 110 \mathrm{Kv}, \mathrm{FSV}: 5,40 \mathrm{~mA}$, SSV: $110 \mathrm{Kv}$, SSV:0,50mA, detector field 12", exposure time: $1,8 \mathrm{~s}, \mathrm{mAs}: 4,80$. (Figure 1)

\section{Case 2}

A woman aged 67 diagnosed with osteoporosis taking bisphophonates treatment developed stage 3 of BONJ (Exposed necrotic bone associated with necrotic bone and osteolysis in the radiological findings) located in the front side of mandibular arch. The radiological protocol of CBCT included the following data: axial thicknes $0,500 \mathrm{~mm}$, FSV:110KV, FSV: 2,70Ma,SSV; 110K, SSV:0,5 mA, detector field: 12 ", exposure time :3,6, mAs:5,36. (figure 2) 

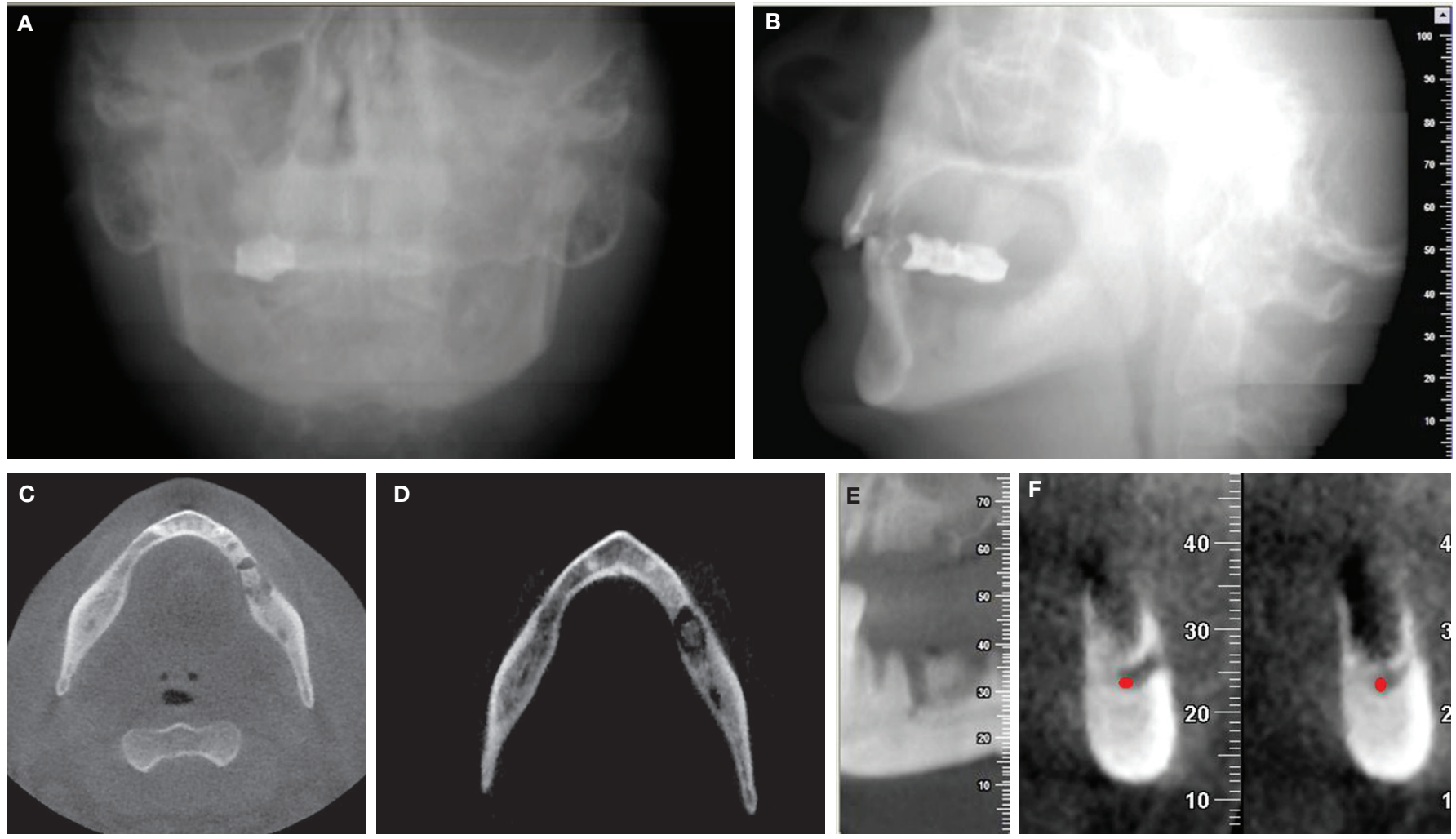

Fig.1. Radiological findings of BONJ located on the lateral left side of the mandibular arch. A. Radiographic skull in frontal view. B. Radiographic skull in lateral view. C.Axial section of the mandibular arch with structural alteration of trabecular bone from initial change in thickness and mineral content of the trabeculae to the formation of microlacunae. D. The location of necrotic site of bone in the lateral left side of the mandibular arch with cortical bone erosion, osteosclerosis and more than $15 \mathrm{~mm}$ sequestrum. E.Axial Panoramic view of the osteonecrosis. F.Sequence of cross action view, the relationship of BONJ with mandibular canal(red point is mandibular canal and the arrow shows the location of osteonecrosis.
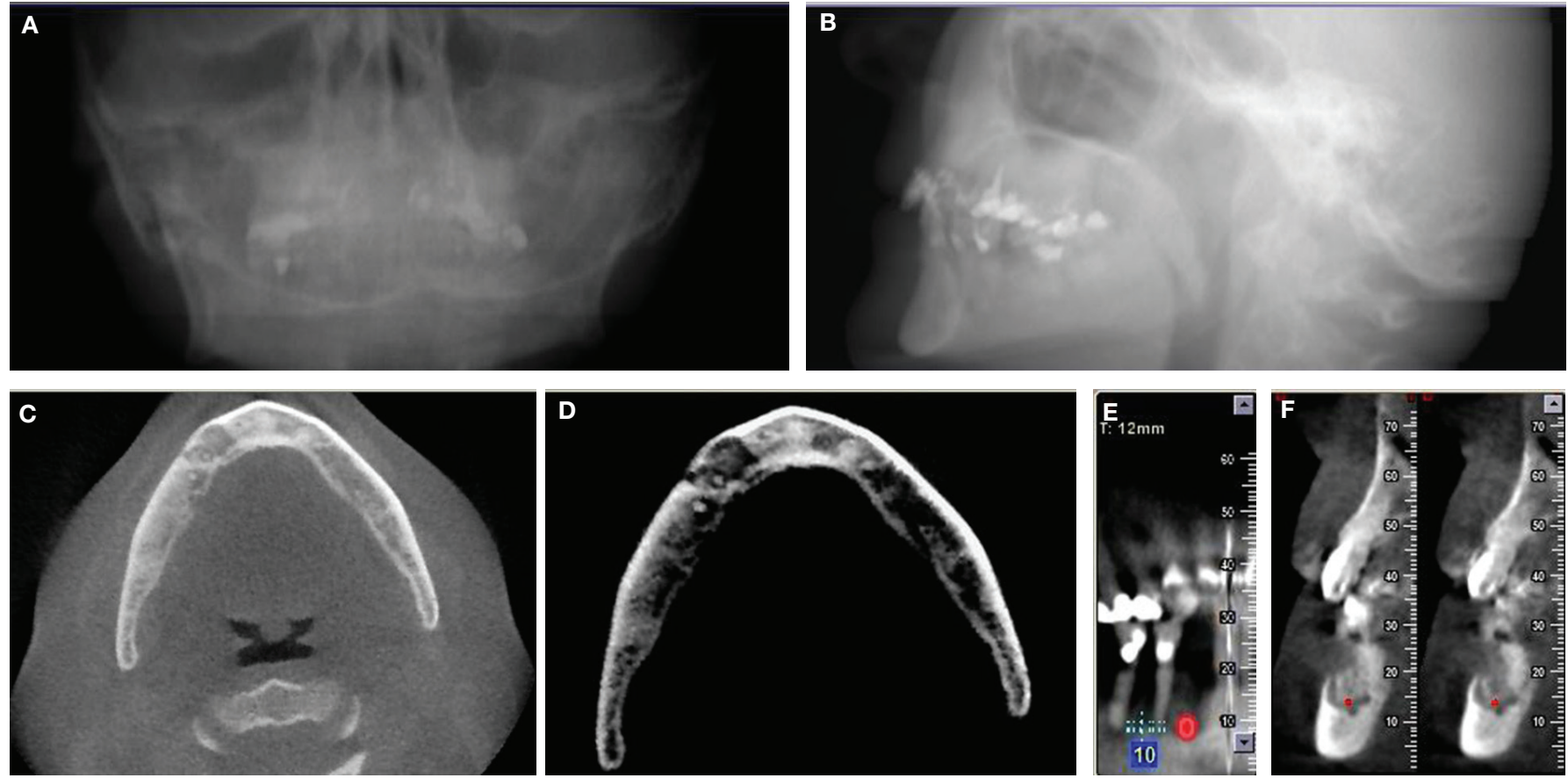

Fig.2. Radiological findings of BONJ located in the front side of mandibular arch. A. Radiographic skull in frontal view. B. Radiographic skull in lateral view. C. Axial section of the mandibular arch with structural alteration of trabecular bone from initial change in thickness and mineral content of the trabecular to the formation of microlacunae. D. The location of the necrotic site of bone in the frontal side of the manibular arch with cortical bone erosion, osteosclerosis and more than $15 \mathrm{~mm}$ sequestrum. E.Axial panoramic view of the osteonecrosis. F.Sequence of cross action view , the relationship of BONJ with mental foramen (red point is the mental foramen and the arrow shows the location of osteonecrosis).

\section{Case 3}

A man aged 73 diagnosed with prostate adenoma under bisphosphonate therapy developed stage 1 of BONJ (Table
I -Asymptomatic exposed necrotic bone or single intraoral fistula and inhomogeneous bone structure in the radiological findings) located mostly in the front side of the mandib- 

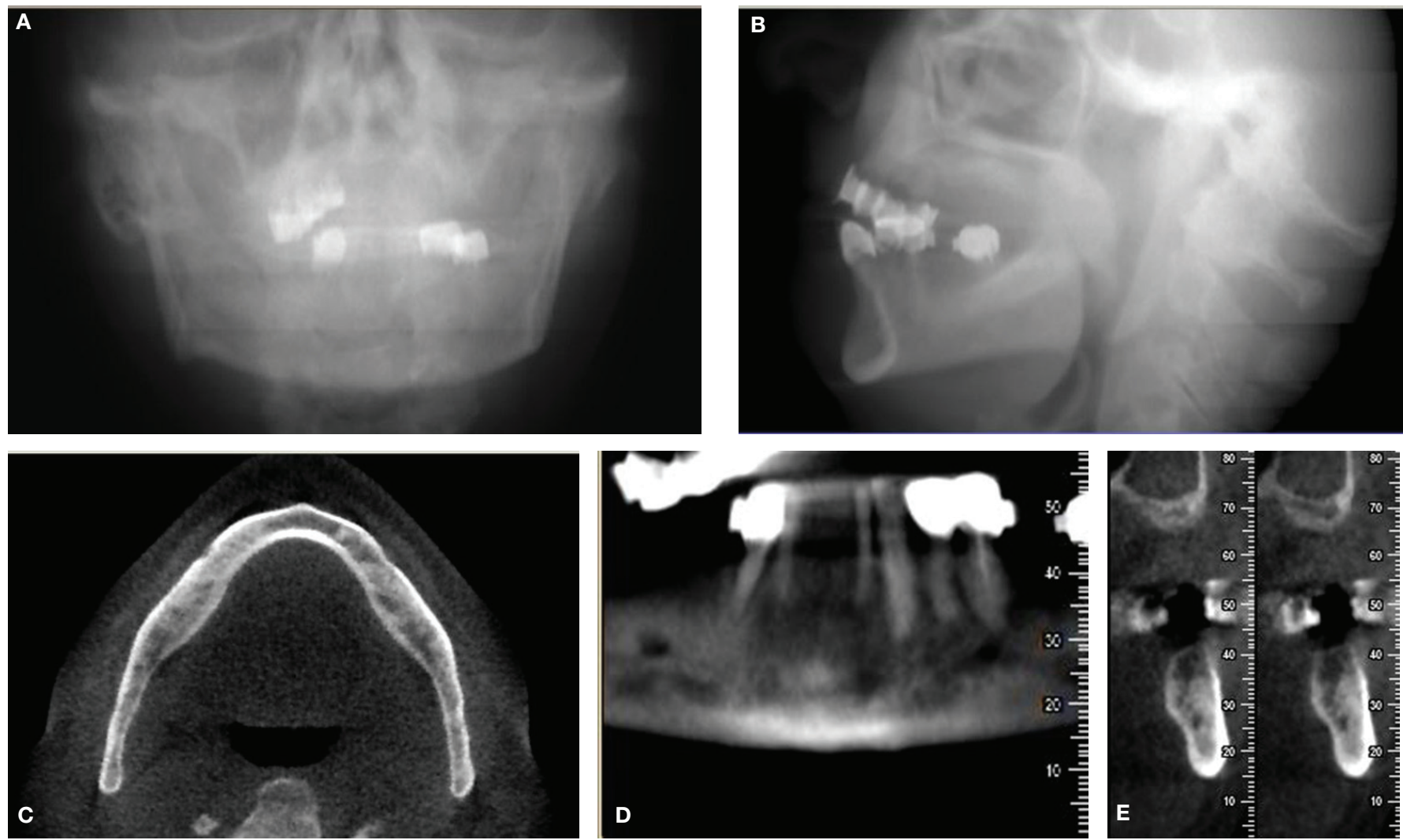

Fig.3. Stage 1 Asymptomatic exposed necrotic bone. A. Radiographic skull in frontal view. B. Radiographic skull in lateral view. C.Axial section of the mandibular arch showing diffuse osteolysis. D.Axial panoramic view -presence of cortical bone erosion and less than $15 \mathrm{~mm}$ sequestrum located in chin area. E.The location of Stage 1 of BONJ from Cross action sequence view.

ular arch. In this case, the radiological protocol of CBCT included the following: axial thickness $0,500 \mathrm{~mm}$, FSV: 110KV, FSV: 3,10Ma, SSV; 110KV, SSV:0,50mA, detector field 12", exposure time 3,6 s, mAs: 6,20 (figure 3).

\section{Results}

A retrospective study was carried out between 2010 - 2012 in patients under therapy with i.v. bisphosphonates according to gender and group of ages; it showed that the prevalence of osteonecrosis of mandibular growth is higher in women than in men (Figure 4). This is due to hormonal factor, women are more affected in this regard [15]. More over, in our study, the prevalence of osteonecrosis of the jaw in appearance depending on age factor which occurs more often between age 52-59 and 73-80 years (Figure5).

\section{Discussion}

Intra-oral radiographs, including periapical, bitewing andocclusal projections are the main and often the only imaging technique required for much dental pathology. Normally, these are reviewed and performed by the general dentist. The radiologist practitioner should be aware that intra-oral radiographs are beeing performed without intensifying screens, and this results in having higher spatial resolution (of the order of 20 line pairs per millimetre (lp/ $\mathrm{mm}$ )) than panoramic radiographs (about $5 \mathrm{lp} / \mathrm{mm}$ ) [22]. The higher spatial resolution allows detection of small carious lesions and periapical lucencies which, usually, may

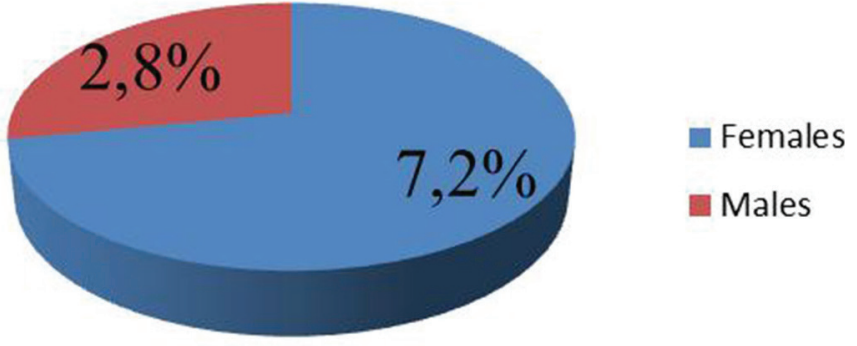

Fig. 4. Distribution on gender

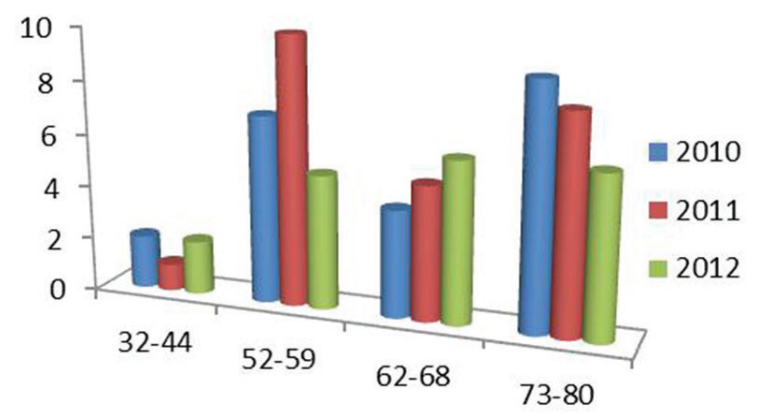

Fig. 5. Distribution on group of ages and the years of study

not always be detected with dental panoramic tomography. Cone-beam computed tomography (CBCT) uses a coneshaped X-ray beam (unlike the fan-shaped X-ray beam used in conventional CT), to acquire projection data into a flat detector, during a single $360^{\circ}$ rotation, from which a volu- 
metric data set is reconstructed using algorithms similar to those used in conventional CT. It results in a lower radiation dose than conventional CT, but it suffers from significant image noise, and is not suitable for softtissue assessment. CBCT is capable of higher spatial resolution (with isotropic voxels as small as $0.125 \mathrm{~mm} 3$ ) than conventional $\mathrm{CT}$. As with conventional CT, the volume data set can be used to create multiplanar and three-dimensional reconstructions. CBCT units are generally cheaper and smaller than conventional CT scanners, and the patient's position is upright, similar to the position in a dental panoramic tomography unit. Thick multiplanar reconstructions can be used to produce lateral and frontal cephalometric images (without distortion or magnification) for orthodontic assessment. Some current CBCT units are also capable of acquiring panoramic tomograms and cephalometric projections directly, as the one used in our study. One important disadvantage of cone beam tomographies is not providing the appropiate images for analysing lamina dura [17], in our cases beeing a very important factor in diagnosis and treatment of BONJ.

The main limitation we had in this study was the fact that the underlying disease, the type of bisphosphonates taken, dose and duration of therapy were not known. As the study was waived of consent, we could not collect data from medical records to obtain such clinical information.

\section{Conclusion}

Despite efforts to minimize risk and prevent occurrence, BONJ will continue to be diagnosted and clinical challenge facing oral health care specialists, oncologists and their patients. As we start to investigate the underlying pathology and biology of BONJ, the prospective epidemiological and interventional trials, the characterization of the basic clinical, biochemical, and radiographic features will be critical. That beeing said, the incidence of $\mathrm{BONJ}$ in cancer patients is approximately 5\% (Bamias et al, 2005; Durie et al, 2005; Zervas et al, 2006; Murad et al, 2007) [22], precise documentation and reporting of retrospective data will continue to provide significant datas into this emerging oral disease. The multitude of complications due to treatment with bisphosphonates bind to an early and specialized therapeutic approach. Radiological examinations is a first choice in the detection and early diagnosis of osteonecrosis of the jaw, patients requiring a permanent supervision by the physician and dentist.

\section{Acknowledegements}

I would like to give many thanks to the Maxilo-Facial Surgery II group for supporting and colaboration in this retrospective study.

\section{References}

1. Hamada H, Matsuo A, Koizumi T, Satomi T, Chikazu D. A simple evaluation method for early detection of bisphosphonaterelated osteonecrosis of the mandible using computed tomography. Journal of Cranio-MaxilloFacial Surgery.2014:1-5.

2. Malden N, Pai AY. Oral bisphosphonate associated osteonecrosis of the jaws: three case reports. British Dental Journal. 2007;203(2):93-96.

3. Graham R, Russell G. Bisphosphonates: Mode of Action and Pharmacology. Pediatrics, 2007;119 (suppl. 2):150-162.

4. Yamashita J, McCauley LK. Antiresorptives and Osteonecrosis of the Jaw. J Evid Base Dent Pract. 2012;S1:233-247.

5. Sharma D, Ivanovski S, Slevin M, et al. Bisphosphonate-related osteonecrosis of jaw (BRONJ): diagnostic criteria and possible pathogenic mechanisms of an unexpected anti-angiogenic side effect. Vascular Cell. 2013;5(1):1-8.

6. Petcu EP, Ivanovski S, Wright RG, Slevin M, Miroiu R, Brinzaniuc K. Bisphosphonate-related osteonecrosis of jaw (BRONJ): an antiangiogenic side-effect? Diagnostic Pathology. 2012;7(78):1-3.

7. Sarasquete ME, Gonzalez M, San Miguel JF, Garcia-Sanz R. Bisphosphonate-related osteonecrosis: genetic and acquired risk factors. Oral Dis. 2009;15:382-387.

8. Arce K, Assael LA, Weissman JL, Markiewicz MR. Imaging Findings in Bisphosphonate-Related Osteonecrosis of Jaws. American Association of Oral and Maxillofacial Surgeons. 2009:76-78.

9. Ferrara F, Califano L. Current perspectives in the management of patients with drug-induced osteonecrosis of the maxilla: experience of the school of Naples. BMC Geriatrics. 2010;10(supl.10):A56.

10. Torres SR, Chen CSK, Leroux BG, Lee PP, Hollender LG, Schubert MM. Fractal dimension evaluation of cone beam computed tomography in patients with bisphosphonate-associated osteonecrosis. Dentomaxillofacial Radiology. 2011;40:501-505.

11. Stockmann P, Hinkmann FM, Lell MM, et al. Panoramic radiograph, computed tomography or magnetic resonance imaging. Which imaging technique should be preferred in bisphosphonate-associated osteonecrosis of the jaw? A prospective clinical study. Clin Oral Invest. 2010;14:311-317.

12. Otto S, Sotlar K, Ehrenfeld M, Pautke C. Osteonecrosis of the jaw as a possible rare side effect of annual bisphosphonate administration for osteoporosis: A case report. J Med Case Rep. 2011 Sep 23;5:477

13. Vogt-Ferrier NB, Hugentobler $M$, Uebelhart $B$, Tramèr $M$, Rollason V. Interventions for treating osteonecrosis of the jaw bones associated with bisphosphonates (Protocol). Cochrane Database of Systematic Reviews 2010, Issue 4. Art. No.: CD008455. DOI: 10.1002/14651858.CD008455.

14. Wong YK. Bisphosphonate-related osteonecrosis of the jaws - Report of 2 cases and strategies on prevention and management. Quintessence Int. 2008 Mar;39(3):195-201.

15. Agrillo A, Filiaci F, Ramieri V, et al. Bisphosphonate-related osteonecrosis of the jaw (BRONJ): 5 year experience in the treatment of 131 cases with ozone therapy. European Review for Medical and Pharmacological Sciences. 2012;16:1741-1747.

16. Boeddinghaus R, Whyte A. Current concepts in maxillofacial imaging. European Journal of Radiology. 2008;66:396.

17. Torres SR, Chen CSK, Leroux BG, Lee PP, Hollender LG, Schubert MM. Fractal dimension evaluation of cone beam computed tomography in patients with bisphosphonate-associated osteonecrosis. Dentomaxillofacial Radiology. 2011;40:504.

18. Conte-Neto N, Bastos AS, Marcantonio RA, Junior EM. Epidemiological aspects of rheumatoid arthritis patients affected by oral bisphosphonaterelated osteonecrosis of the jaws. Head Face Med. 2012 Mar 1;8:5.

19. Sanna G, L Preda, R Bruschini, et al. Bisphosphonates and jaw osteonecrosis in patients with advanced breast cancer. Annals of Oncology. 2006;17:1512-1516.

20. del Castillo Pardo de Vera JL, García de Marcos JA, Rodríguez SA, Arenas MG, Calderón Polanco J. Osteonecrosis de los maxilares asociada al empleo de bifosfonatos Bisphosphonate-associated osteonecrosis of the jaw. Rev Esp Cir Oral y Maxilofac. 2007;29(5):295-308.

21. Wilde F, Heufelder M, Lorenz K, et al. MSCS, Rostock. Prevalence of cone beam computed tomography imaging findings according to the clinical stage of bisphosphonate-related osteonecrosis of the jaw. december 2012;114(6):805.

22. Treister N, Sheehy N, Bae EH, Friedland B, Lerman M, Woo S. Dental panoramic radiographic evaluation in bisphosphonate-associated osteonecrosis of the jaws. Oral Diseases. 2009;15:90-91. 